\title{
CEOs from Orthopaedic Centers Worldwide Meet to Discuss Common Challenges: 2010 Annual Meeting of the International Society of Orthopaedic Centers
}

\author{
Laura Robbins, DSW • Louis A. Shapiro, FACHE • Matthias P. Spielmann, MHA - Giovanni Baldi, MD
}

Received: 11 October 2010/Accepted: 10 December 2010/Published online: 11 February 2011

(C) The Author(s) 2011. This article is published with open access at Springerlink.com

\begin{abstract}
The International Society of Orthopaedic Centers was formed in 2006 as a think tank that would bring together thought leaders in orthopaedic surgery from major orthopaedic academic centers around the world. The Society's mission is to share knowledge and strategies, improve patient care, and foster clinical, educational, and scientific collaboration. As the Society's agendas developed, the members recognized that many of their aims intersected with those of hospital leadership. Thus, CEOs from member centers were invited to join their physician colleagues at the 2010 meeting in Bologna, Italy in order to explore solutions to administrative challenges related to patient care, volume growth, and costs. This paper describes the dialogue that took place at the meeting.
\end{abstract}

Keywords hospital CEOs.

International Society of Orthopaedic Centers (ISOC). orthopaedic surgeons

Each author certifies that he or she has no commercial associations (e.g., consultancies, stock ownership, equity interest, patent/licensing arrangements, etc.) that might pose a conflict of interest in connection with the submitted article.

L. Robbins, DSW $(\bowtie) \cdot$ L. A. Shapiro, FACHE

Hospital for Special Surgery,

535 East 70th Street,

New York, NY 10021, USA

e-mail: robbinsl@hss.edu

M. P. Spielmann, MHA

Schulthess Klinik,

Lëngghalde 2,

3003, Zurich, Switzerland

G. Baldi, MD

Istituto Ortopedico Rizzoli,

via Giulio Cesare Pupilli, 1,

40136, Bologna, Italy

\section{Introduction}

Hospital CEOs worldwide are grappling with similar challenges, including issues related to patient safety, quality, best practices, and how to best minimize costs [1-4]. CEOs in the United States have the opportunity to meet and seek solutions regularly, for example, at seminars conducted by the American College of Healthcare Executives. The opportunities to communicate internationally are fewer, even though hospitals in many countries share the same challenges related to balancing best patient care with costeffective measures.

In addition, enormous changes have taken place in the patterns of musculoskeletal diseases over the last decades, including a significant increase in degenerative disease due to an aging population $[5,6]$. At the same time, advances in surgical techniques have occurred, many fuelled by high technology and the major research efforts in biomechanics, biomaterials, and electrophysiology that have added to the pool of orthopaedic knowledge. In 2006, the International Society of Orthopaedic Centers (ISOC; www.isocweb.org) was formed in response to the need to share these advances on a global basis [7]. The Society presently has 14 participating centers from 11 countries. The members come from large orthopaedic departments within general hospitals connected to an academic center, or from academically oriented specialty orthopaedic hospitals. ISOC's mission is to promote scientific, clinical, and educational collaboration among participating institutions; to improve the quality of patient care through sharing of clinical pathways and treatments; to collaborate on academic programs through the exchange of residents, fellows, and faculty; and to share cost-effective measures that will ensure economic success for major orthopaedic programs.

As these aims were elaborated over time, the Society realized that many of its goals overlapped with those of 
hospital leadership. To afford CEOs the opportunity to look at the practices of other institutions worldwide, and to share solutions, the ISOC Board of Directors decided to invite the member centers' CEOs to the ISOC annual meeting that took place at the Istituto Ortopedico Rizzoli in Bologna, Italy, April 29 through May 1, 2010. Thirteen CEOs/ managers from 11 institutions accepted the invitation.

\section{Attending CEOs}

Chile Alejandro de Marinis, MD, Deputy Director Clinica Alemana de Santiago (Santiago)

Germany Sibylle Stauch-Eckmann, Managing Director Endo Klinik (Hamburg)

Italy Elena Botinelli, CEO IRCCS

Istituto Ortopedico Galeazzi (Milan)

Italy Giovanni Baldi, MD, CEO

Istituto Ortopedico Rizzoli (Bologna)

Mexico Luis Ibarra, MD, General Director

Maricela Verdejo, $\mathrm{PhD}$,

Director of Administration

Instituto Nacional De Rehabilitacion

(Mexico City)

Netherlands Peter Hoppener, CEO

Sint Maartenskliniek (Nijmegen)

Sweden Magnus Eneroth, MD, PhD, CEO

Pelle Gustafson, $\mathrm{MD}, \mathrm{PhD}, \mathrm{COO}$

Skåne University Hospital (Lund)

Switzerland Matthias P. Spielmann, MHA, CEO

Schulthess Klinik (Zurich)

United States Paige LeMay, MHA, COO

Campbell Clinic (Germantown, TN)

United States Louis A. Shapiro, FACHE, President and CEO Hospital for Special Surgery

(New York, NY)

United States Michael Rock, MD, Medical Director/CMO Mayo Clinic Hospitals (Rochester, MN)

CEO invitees from three additional institutions were unable to attend this session: University Hospitals Leuven in Belgium, Peking Union Medical College Hospital in China, and the Royal National Orthopaedic Hospital in the United Kingdom.

\section{The First CEO Meeting, 2010}

At the first session, the CEOs gave Power Point presentations setting their institutions in context by describing the health care systems of their countries and then characterizing their centers in terms of history, size, areas of specialization, research efforts, teaching programs, current challenges, initiatives under way, and strategic plans. Among the challenges outlined were the cost of care, declining reimbursement, the need to improve financial performance (by internal reorganization, greater efficiency, etc.), the vulnerability of a specialized hospital, long wait times for surgery, constraints on physical expansion (in terms of both space and costs), shortage of skilled staff, the need for more up-to-date technology, the rising costs of medical devices, greater use of physician extenders, and maintaining research and academic excellence despite a decline in funding.

Three general topics were chosen from the list of challenges submitted before the meeting, in an attempt to explore themes that would be valid for different global institutions. The next three sessions were devoted to these topics: growth, capacity management, and economic challenges. These topics had been carefully chosen before the meeting in Bologna in order to facilitate focused, fruitful discussions around themes that would be valid globally, as health systems and policies vary from country to country. A decision was made to address these policy differences specifically in future meetings. (The ISOC physicians group, which has now met three times, has found that universal ground could easily be reached on many of their concerns, such as resident education, mapping out areas of research for each center, and collaborating on tissue engineering and analysis of pooled data, for example [8].)

At the open session on the last day, the CEOs reached some general conclusions and explored future directions. They then joined the physicians for a joint discussion intended to span clinical and administrative issues such as the affordability of new technology, retrieval analysis of joint replacement, national registers, and methods of improving musculoskeletal health.

\section{Results: Consensus of the CEO Participants}

The session on growth, facilitated by Louis Shapiro from Hospital for Special Surgery, explored how each institution is addressing growth-related challenges. The group reached the following conclusions:

- The demand for services and therefore growth comes from being the best. Data on patient-centered outcomes and transparency of results will be increasingly important.

- Most of the centers are dealing with the need to expand physical space and capacity. Efficiency and throughput are essential for creating increased capacity.

- Some of the institutions are thinking about replicating business models in other geographic locations. Some possibilities are partnering with other hospitals, off-site programs, or additional campuses. There were differences regarding the degree of willingness/ability to partner with other institutions.

Matthias Speilmann from the Schulthess Klinik chaired the session on capacity management issues, which concluded the following:

- Demand exceeds capacity in most institutions.

- Some institutions may need to prioritize patients. 
- Efficiency was again identified as a critical component of creating additional capacity.

Giovanni Baldi from the Istituto Ortopedico Rizzoli led the session on economic challenges, which reached the following conclusions:

- The introduction of new technology needs to be carefully managed, and surgeons should play a leadership role in this effort.

- Physicians should align around the objective of creating value for the patient.

- Administrators should consider the use of "lean healthcare" principles to address economic issues [9]. (Taken from the concept of "lean manufacturing," lean healthcare supplies a set of concepts and tools aimed at eliminating waste. The process begins by defining patient needs and then redesigning the system to meet those needs more efficiently, for example, by decreasing wait time and paperwork, optimizing inventory, and concentrating on staff that perform value-added activities. Ideally, the principles are put in place across the whole institution.)

At the final session, chaired by Alejandro de Marinis from the Clinica Alemana, the group discussed collaboration opportunities for the future, and agreed on the following:

- A number of possible areas for collaboration were identified, the first one being the sharing of best metrics, with a specific focus on physician accountability. A task force of three was set up, to include Pelle Gustafson, Sybille Stauch-Eckmann, and Louis A. Shapiro. Each CEO will partner with a physician from his/her institution.

- Other collaborative areas identified were approaches to efficiency (lean healthcare) and clinical pathways for specific patient types.

- In order to foster further collaboration and dialogue around common areas of interest, mutual visits were suggested to observe operational approaches. (Since the ISOC meeting, a group from the Endo Klinik in Hamburg, Germany has spent a week in the United States visiting both the Mayo Clinic and Hospital for Special Surgery. The group consisted of Sibylle StauchEckmann, Managing Director; Thorsten Gehrke, MD, $\mathrm{PhD}$, Medical Director; Daniel Kendoff, MD, PhD, Associate Professor of Orthopaedic Surgery; and Philip Wettengel, Assistant to the Management.)

- All members felt that the sessions had been beneficial. It was decided to convene again at the next ISOC meeting in the fall of 2011 at Clinica Alemana in Chile and decide at that point whether to continue as a group. Alejandro de Marinis offered to take the lead in planning the meeting, with focused presentations on specific topics of general interest such as medical malpractice.

- It was suggested that the proceedings be written up for submission to a peer-reviewed journal.

\section{CEO and Physician Joint Session}

Louis A. Shapiro summarized the CEO meetings for the benefit of the physicians (the information presented in this paper). The Joint Session gave the CEOs the opportunity to hear summaries (in areas of mutual interest) from the physician group, which had been meeting concurrently.

The physician group, composed of 37 members from 11 institutions, met in three sessions on previously chosen topics: basic science, joint arthroplasty, and spine/ankle/ cerebral palsy. The task forces set up at the 2008 annual meeting also presented reports on education/fellowship, outcomes/clinical studies/registries, implant retrieval, and information exchange/website.

The traveling fellowships are to begin in September 2011. Nine ISOC institutions will be sponsors: Clinica Alemana, Endo Klink, Hospital for Special Surgery, Instituto Nacional De Rehabilitacion, Istituto Ortopedico Galeazzi, Istituto Ortopedico Rizzoli, Skåne University Hospital, Schulthess Klinik, and the Royal National Orthopaedic Hospital.

The Outcomes/Clinical Studies/Registries Task Force reported that a steering committee will move forward on the first joint pilot projects, by identifying sites with common interests and infrastructures, developing a joint study protocol that is applicable to all sites, and setting up a harmonization process and a method of ensuring data quality (for example, according to the requirements of government bodies from the various countries). The protocol will include core data sets, inclusion and exclusion criteria, intervals, and follow-up schemes, as well as legal aspects and joint application for funding.

The Implant Retrieval Task Force is in the process of identifying timely, high-impact research questions centered around device technology, surveying ISOC members using implant retrieval to determine what data are available, and establishing the policies needed for conducting collaborative research. Preliminary discussions have identified two pilot projects:

1. Metal-on-metal bearings for total hip replacement and hip resurfacing, which have received little clinical evaluation compared with metal-on-polyethylene bearings. What are the modes of wear and surface damage? How are the presence and severity of these modes affected by patient, surgical, and design factors?

2. Rotating platform (intended to reduce wear) versus fixed-bearing total knee replacements. A recent Hospital for Special Surgery study has shown considerable wear at both bearing surfaces. What is the relation between rotating platform surface damage and actual wear? How do rotating platform designs compare with similar fixedbearing designs in terms of surface damage and wear to the femorotibial articulating surfaces as well as total damage and wear?

The Information Exchange/Website Task Force reported that the website, the purpose of which is to publicize the 
existence of ISOC and foster communication and collaboration among the centers, has been successfully launched (www.isocweb.org). Both the physicians and the CEOs discussed use of the website to share best practices at their academic centers.

\section{Summary}

In 2009, ISOC became legally incorporated and has three new members this year, Sint Maartenskliniek (Netherlands), Skåne University Hospital (Sweden), and the Mayo Clinic (United States). Three other institutions (from Australia, South Africa, and Canada) have applied for membership, and centers in other countries are being considered for invitations. The CEO participants agreed that their dialogues had been illuminating. The next steps would involve identifying common crosscultural issues (that would also apply to their different health systems), to establish a matrix by which to define best practices and continuing to meet in order to determine common administrative issues related to these best practices. As was the case with the orthopaedic surgeons when they first met (in 2006), the CEOs agreed to allow their issues to evolve over time at future meetings. The fourth ISOC meeting will take place in Santiago, Chile, at Clinica Alemana, on October 13-15, 2011.
Acknowledgments The authors thank Phyllis Tower for help in manuscript preparation.

Open Access This article is distributed under the terms of the Creative Commons Attribution Noncommercial License which permits any noncommercial use, distribution, and reproduction in any medium, provided the original author(s) and source are credited.

\section{References}

1. American College of Healthcare Executives announces top issues confronting hospitals. www.ache.org/Pubs/Releases/ 2010/TopIssues_2010.pdf. Accessed 2 Jul 2010.

2. Bohmer RMJ. Fixing health care on the front lines. Harv Bus Rev 2010; 88:62-69.

3. Herzlinger RE. Hospital for Special Surgery (C): Continuing challenges of growth. Cambridge, MA; Harvard Business Publishing for Educators; January 25, 2010.

4. Levin-Scherz J. What drives high health care costs - and how to fight back. Harv Bus Rev 2010; 88:72-73.

5. Edwards TB. The globalization of orthopaedics. Orthopaedics 2001; 24:1032-1036.

6. Leong JCY. Should orthopaedics be globalised? Int Orthop 2005; 29:201-204.

7. Sculco TP, Klinghoffer IP. Global musculoskeletal health: inaugural meeting of the International Society of Orthopaedic Centers. HSSJ 2008; 5:24-26.

8. Sussmann PS, Simmen BR, Goldhahn J, ISOC participants, Sculco TP (2010) Challenges for large orthopaedic hospitals worldwide - an ISOC position statement. HSS J 2010; 6:57-60.

9. De Koning H, Verver JPS, van den Heuvel J, Bisgaard S, Does RJMM. Lean Six Sigma in healthcare. J Healthc Qual 2006; 206:174. 